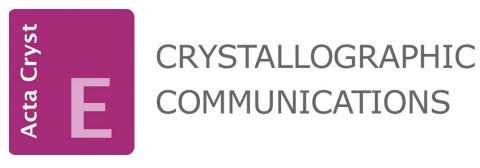

ISSN 2056-9890

Received 12 October 2020

Accepted 23 October 2020

Edited by W. T. A. Harrison, University of Aberdeen, Scotland

Keywords: crystal structure; tetracyanonickelate; $\mathrm{N}$-(pyridin-2-yl)pyridinium-2-amine; hydrogen bonding; Hirshfeld surface analysis; crystal structure.

CCDC reference: 2040378

Supporting information: this article has supporting information at journals.iucr.org/e

\section{Synthesis, crystal structure and Hirshfeld surface analysis of bis\{2-[(pyridin-2-yl)amino]pyridinium\} tetracyanonickelate(II)}

\author{
Zouaoui Setifi, ${ }^{\text {a,b }}$ Hela Ferjani, ${ }^{c *}$ Fatima Setifi, ${ }^{a *}$ Safa Ezzine ${ }^{\mathrm{d}}$ and Mohammed Hadi \\ Al-Douh ${ }^{\text {e }}$
}

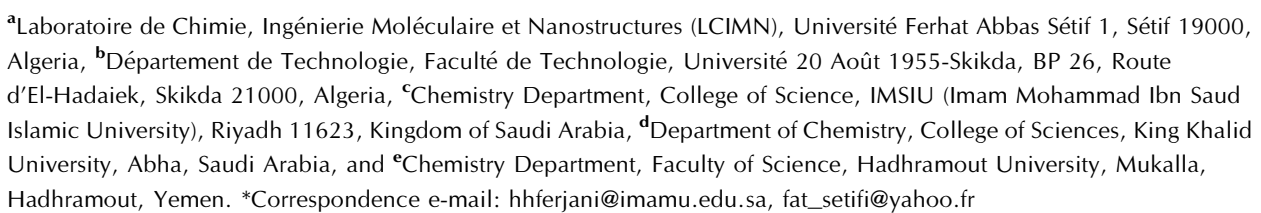

In the title molecular salt, $\left(\mathrm{C}_{10} \mathrm{H}_{10} \mathrm{~N}_{3}\right)_{2}\left[\mathrm{Ni}(\mathrm{CN})_{4}\right]$, the dihedral angle between the pyridine rings in the cation is $1.92(13)^{\circ}$ and the complete anion is generated by a crystallographic centre of symmetry. An intramolecular $\mathrm{N}-\mathrm{H} \cdots \mathrm{N}$ hydrogen bond occurs in the cation, which closes an $S(6)$ ring. In the crystal, the components are linked by $\mathrm{N}-\mathrm{H} \cdots \mathrm{N}$ and weak $\mathrm{C}-\mathrm{H} \cdots \mathrm{N}$ hydrogen bonds, which generate chains propagating in the [101] direction. Weak aromatic $\pi-\pi$ stacking interactions are also observed. A Hirshfeld surface analysis and twodimensional fingerprint plots indicate that the most important contact types in the crystal packing are $\mathrm{N} \cdots \mathrm{H} / \mathrm{H} \cdots \mathrm{N}, \mathrm{C} \cdots \mathrm{H} / \mathrm{H} \cdots \mathrm{C}$ and $\mathrm{H} \cdots \mathrm{H}$ with contributions of $37.2,28.3$ and $21.9 \%$, respectively.

\section{Chemical context}

Transition-metal coordination compounds, where $\mathrm{CN}^{-}$ligands play the main structure-forming role, so-called cyanocarbanion or cyanometallate complexes, have been the subject of interest for many years, in particular due to their magnetic properties (Ferlay et al., 1995; Bretosh et al., 2020; Benmansour et al., 2012; Setifi et al., 2009; Yuste et al., 2009; Addala et al., 2015), including spin-crossover behavior (Benmansour et al., 2010; Yoon et al., 2011). The square-planar tetracyanonickelate(II) anion $\left[\mathrm{Ni}(\mathrm{CN})_{4}\right]^{2-}$ has proved to be very versatile and diverse in both coordination chemistry and magnetism.

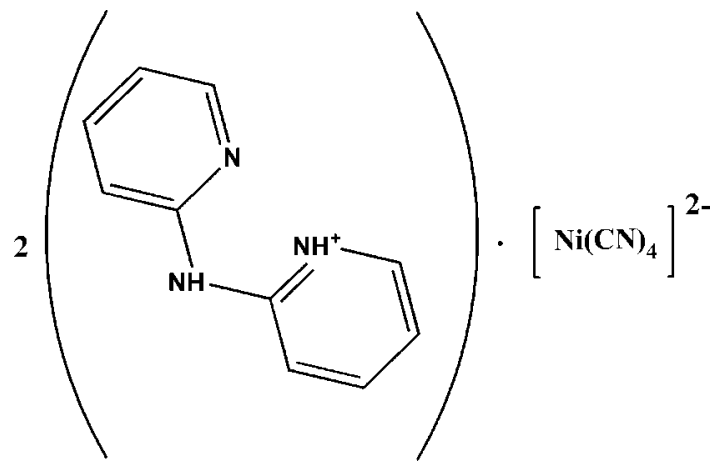

We have been interested in using the tetracyanonickelate(II) anion in combination with other chelating or bridging neutral co-ligands to explore their structural features 
and properties relevant to the field of molecular materials exhibiting the spin-crossover phenomenon (Setifi et al., 2013, 2014; Kucheriv et al., 2016). During the course of attempts to prepare such complexes with 2,2'-dipyridylamine (dpa), we isolated the title molecular salt, (I), whose molecular and supramolecular structure is described herein.

\section{Structural commentary}

The asymmetric unit of (I) contains one $\left(\mathrm{C}_{10} \mathrm{H}_{10} \mathrm{~N}_{3}\right)^{+}$cation and one half of a $\left[\mathrm{Ni}(\mathrm{CN})_{4}\right]^{2-}$ anion (Fig. 1). The $\mathrm{C}-\mathrm{N}$ and $\mathrm{C}-\mathrm{C}$ bonds lengths in the cation vary from 1.340 (3) to 1.383 (3) $\AA$ and from 1.346 (4) to 1.402 (3) $\AA$, respectively. The $\mathrm{C}-\mathrm{N}-\mathrm{C}$ bond angles range from 117.8 (2) to $129.7(2)^{\circ}$ and the $\mathrm{N}-\mathrm{C}-\mathrm{C}$ angles range from 119.0 (2) to $123.4(2)^{\circ}$. The dihedral angle between the C3-C7/N4 and C8-C12/N5 rings is $1.92(13)^{\circ}$. These data are comparable to those found for other compounds containing dpa as an organic template (Bowes et al., 2003; Willett, 1995). In the cation, the pyridyl nitrogen atoms are arranged on both sides of the central N3 atom and assume a cis conformation (Fig. 1). The $\left(\mathrm{C}_{10} \mathrm{H}_{10} \mathrm{~N}_{3}\right)^{+}$ cation is monoprotonated at the pyridyl-N4 atom, which leads to the the formation of a short and presumably strong intra-

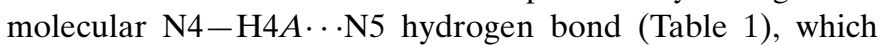
generates an $S(6)$ ring (Fig. 2).

The $\mathrm{Ni}^{2+}$ ion of the anion is located on a crystallographic inversion center and coordinates four terminal (non-bridging)

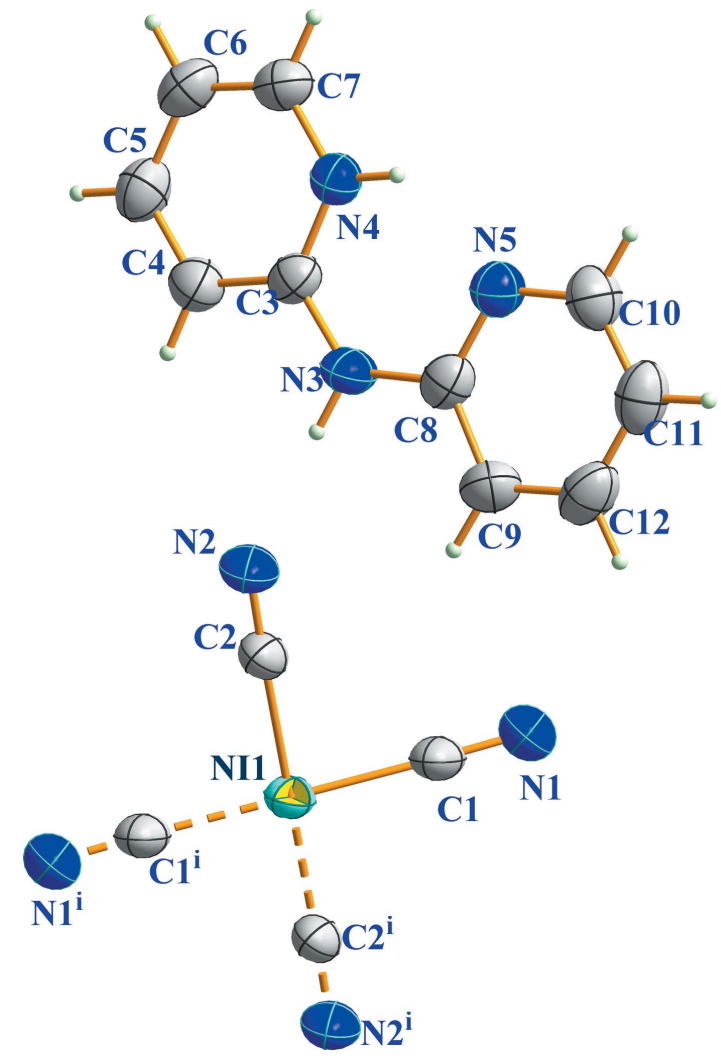

Figure 1

The molecular structure of (I) with displacement ellipsoids drawn at the $50 \%$ probability level. Symmetry code: (i) $-x+1,-y,-z$
Table 1

Hydrogen-bond geometry $\left(\AA{ }^{\circ}\right)$.

\begin{tabular}{lllll}
\hline$D-\mathrm{H} \cdots A$ & $D-\mathrm{H}$ & $\mathrm{H} \cdots A$ & $D \cdots A$ & $D-\mathrm{H} \cdots A$ \\
\hline $\mathrm{N} 3-\mathrm{H} 3 A \cdots \mathrm{N} 2$ & 0.86 & 2.00 & $2.853(3)$ & 172 \\
$\mathrm{~N} 4-\mathrm{H} 4 A \cdots \mathrm{N} 5$ & 0.86 & 1.97 & $2.629(3)$ & 132 \\
$\mathrm{~N} 4-\mathrm{H} 4 A \cdots \mathrm{N} 1^{\mathrm{ii}}$ & 0.86 & 2.41 & $3.055(3)$ & 132 \\
$\mathrm{C} 5-\mathrm{H} 5 \cdots \mathrm{N} 1^{\mathrm{ii}}$ & 0.93 & 2.68 & $3.206(4)$ & 117 \\
\hline
\end{tabular}

Symmetry code: (ii) $-x+1,-y+1,-z+1$

cyanide ligands, exhibiting a square-planar geometry. The bond lengths and angles in the anion are in good agreement with those found in other $\left[\mathrm{Ni}(\mathrm{CN})_{4}\right]^{2-}$ salts (Paharová et al., 2003; Karaağaç et al., 2013).

\section{Supramolecular features}

Fig. 3 shows the packing of (I) in a view along the $b$-axis direction, in which the organic and inorganic ions form chains propagating in the [101] direction linked by $\mathrm{N}-\mathrm{H} \cdots \mathrm{N}$ and $\mathrm{C}-\mathrm{H} \cdots \mathrm{N}$ hydrogen bonds. The pyridinium $\mathrm{N} 4$ atom in the cation, as well as forming the intramolecular hydrogen bond described above, acts as donor to the cyanate $\mathrm{N}$ atom in the anion, in an $\mathrm{N} 4-\mathrm{H} 4 A \cdots \mathrm{N} 1^{\mathrm{ii}}$ [symmetry code: (ii) $-x+1$, $-y+1,-z+1)$ link (Table 1 ). The secondary amino group $(\mathrm{N} 3 H)$ forms a strong $\mathrm{N} 3-\mathrm{H} 3 A \cdots \mathrm{N} 2$ hydrogen bond with a cyano group acceptor and the $\mathrm{H} 3 A \cdots \mathrm{N} 2$ distance is $2.0 \AA$. Fig. 3 shows the parallel offset $\pi$-stacking contacts between pyridyl groups [centroid-centroid distance of 4.3421 (16) ̊] and parallel face-centred $\pi$-stacking interactions between the $S(6)$ centroids and pyridyl groups [centroid-centroid distance

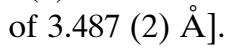

\section{Hirshfeld surface analysis}

Hirshfeld surface calculations (Spackman \& Jayatilaka, 2009) for (I) were performed in order to further characterize the supramolecular association. The Hirshfeld surfaces and twodimensional fingerprint plots (McKinnon et al., 2007) calculated using CrystalExplorer 17.5 (Turner et al., 2017) are shown

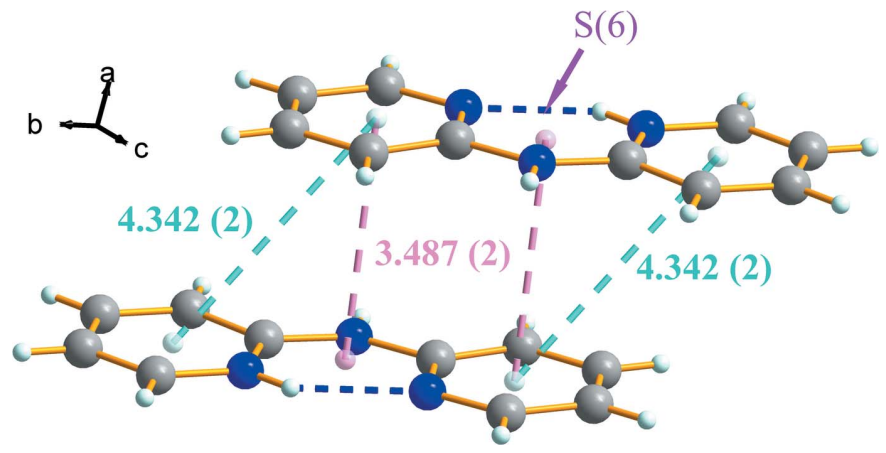

Figure 2

Offset and parallel $\pi-\pi$-stacking interactions (broken lines) in the cationcation chains. 


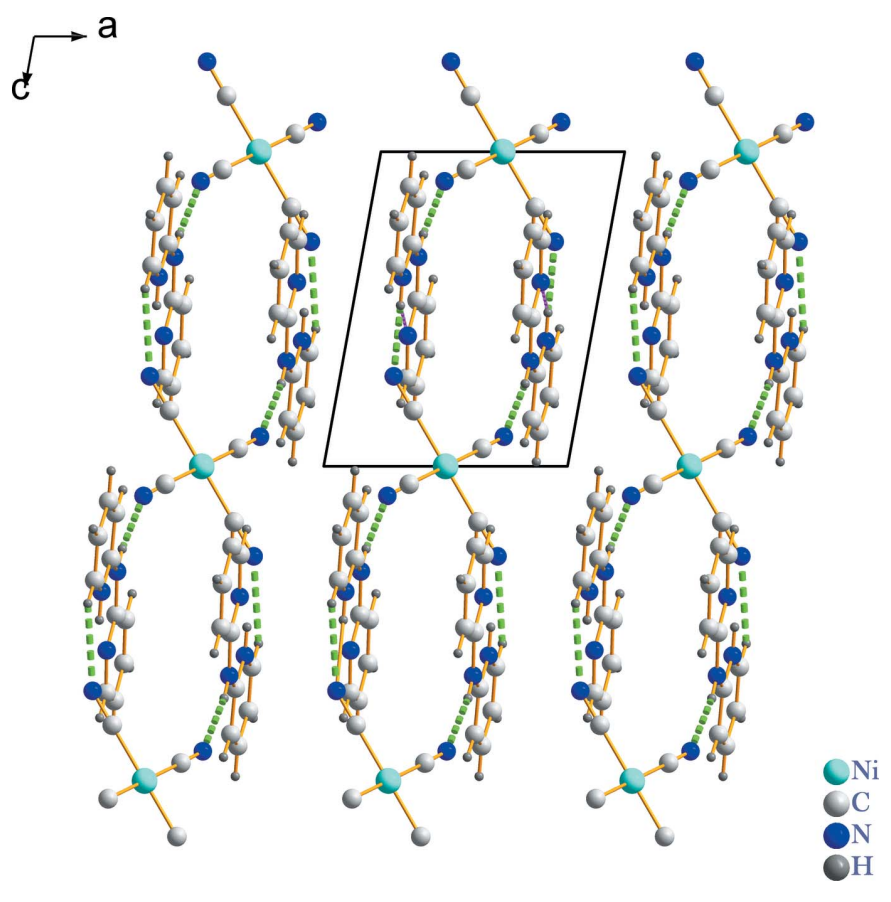

Figure 3

View parallel to the $a c$ plane of the packing in (I) with hydrogen bonds shown as green dashed lines.

in Figs. 4 and 5, respectively. The red spots on the Hirshfeld surface represent strong interaction through $\mathrm{N}-\mathrm{H} \cdots \mathrm{N}$ and $\mathrm{C}-\mathrm{H} \cdots \mathrm{N}$ hydrogen bonding, whereas the blue color represents a lack of interaction. The presence of $\pi-\pi$ stacking interactions is indicated by adjacent red and blue triangles on the shape-index surface (Fig. S1 $a$ in the supporting information). Areas on the Hirshfeld surface with high curvedness (Fig. S1b) can be related to the planar packing arrangement of the cations. The most abundant intermolecular interactions in the crystal packing (Fig. 5) are $\mathrm{N} \cdots \mathrm{H} / \mathrm{H} \cdots \mathrm{N}, \mathrm{C} \cdot \mathrm{H} / \mathrm{H} \cdots \mathrm{C}$ and $\mathrm{H} \cdots \mathrm{H}$ with percentage contributions $37.2,28.3$ and $21.9 \%$, respectively. The presence of weak $\pi-\pi$ stacking interactions between the cationic rings are reflected in the 4.6 and $3.8 \%$ contributions from $\mathrm{C} \cdots \mathrm{C}$ and $\mathrm{C} \cdot \mathrm{N} / \mathrm{N} \cdots \mathrm{C}$ contacts to the Hirshfeld surfaces of the cations. The analysis reveals the lowest contribution of $\mathrm{Ni} \cdot \mathrm{N}(1.7 \%), \mathrm{Ni} \cdot \mathrm{C}(1.3 \%)$ and N. . N $(1.2 \%)$ contacts.

\section{Database survey}

A search of the Cambridge Structural Database (Version 5.41, last update November, 2019; Groom et al., 2016), for the tetracyanonickelate moiety revealed 532 hits. Most of them are complexes of $\left[\mathrm{Ni}(\mathrm{CN})_{4}\right]^{2-}$ anions with different metalligand coordination cations. Salts containing tetracyanonickelate anions and organic cations corresponded to 38 hits.

A compound closely related to the title compound is $\left(\mathrm{C}_{10} \mathrm{H}_{11} \mathrm{~N}_{3}\right) \cdot\left[\mathrm{CuCl}_{4}\right]$ (Willett, 1995; CSD refcode ZAMCEV), which crystallizes in the same space group of $P \overline{1}$. In this compound the cation is diprotonated and the pyridyl nitrogen atoms are in a cis conformation and the pyridine rings are

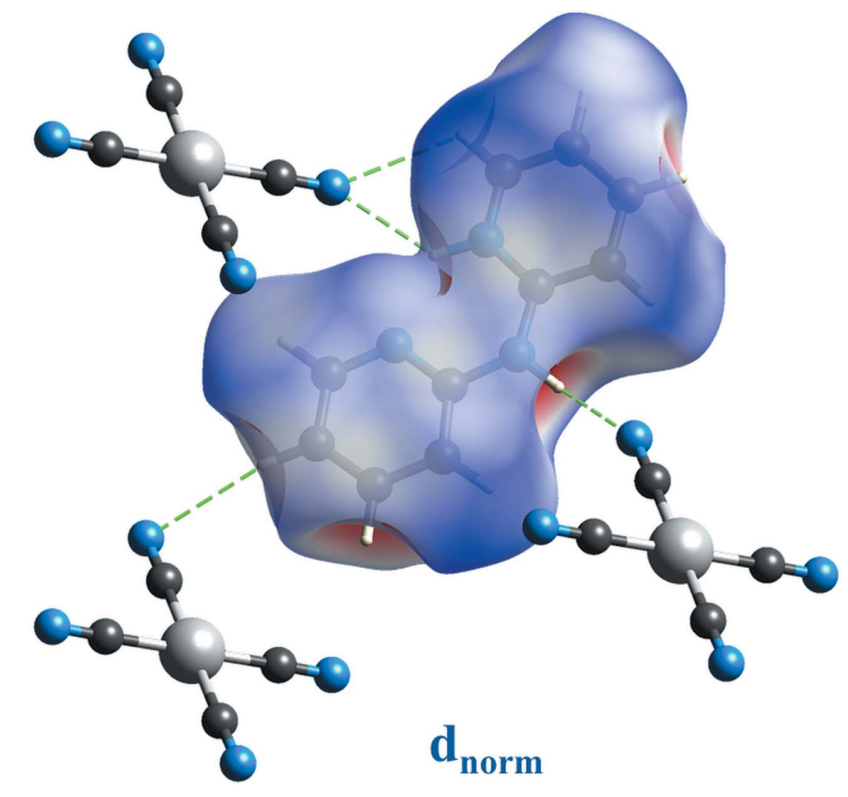

Figure 4

Hirshfeld surface of (I) mapped over $d_{\text {norm }}$.

significantly twisted away from coplanarity. The tetrachlorocuprate anion takes on a squashed tetrahedral geometry.

\section{Synthesis and crystallization}

The title compound was synthesized solvothermally under autogenous pressure using a mixture of iron(II) sulfate heptahydrate $(28 \mathrm{mg}, 0.10 \mathrm{mmol}), 2,2^{\prime}$-dipyridylamine $(17 \mathrm{mg}$, $0.10 \mathrm{mmol}$ ) and potassium tetracyanonickelate(II) $(24 \mathrm{mg}$, $0.10 \mathrm{mmol})$ in mixed solvents of water/ethanol $(3: 1 \mathrm{v} / \mathrm{v}, 20 \mathrm{ml})$. The mixture was sealed in a Teflon-lined autoclave and held at $423 \mathrm{~K}$ for $3 \mathrm{~d}$, and then cooled to room temperature at a rate of $10 \mathrm{~K}$ per hour (yield 27\%). Pale-yellow plates of (I) suitable for single-crystal X-ray diffraction analysis were selected.

\section{Refinement}

Crystal data, data collection and structure refinement details are summarized in Table 2. All $\mathrm{H}$ atoms were positioned geometrically in idealized positions and constrained to ride on their parent atoms, with $\mathrm{C}-\mathrm{H}=0.93$ or $\mathrm{N}-\mathrm{H}=0.86 \AA$, and with $U_{\text {iso }}(\mathrm{H})=1.2 U_{\text {eq }}(\mathrm{C}, \mathrm{N})$.

\section{Funding information}

FS gratefully acknowledges the Algerian Ministère de l'Enseignement Supérieur et de la Recherche Scientifique (MESRS), the Direction Générale de la Recherche Scientifique et du Développement Technologique (DGRSDT) as well as the Université Ferhat Abbas Sétif 1 for financial support. 

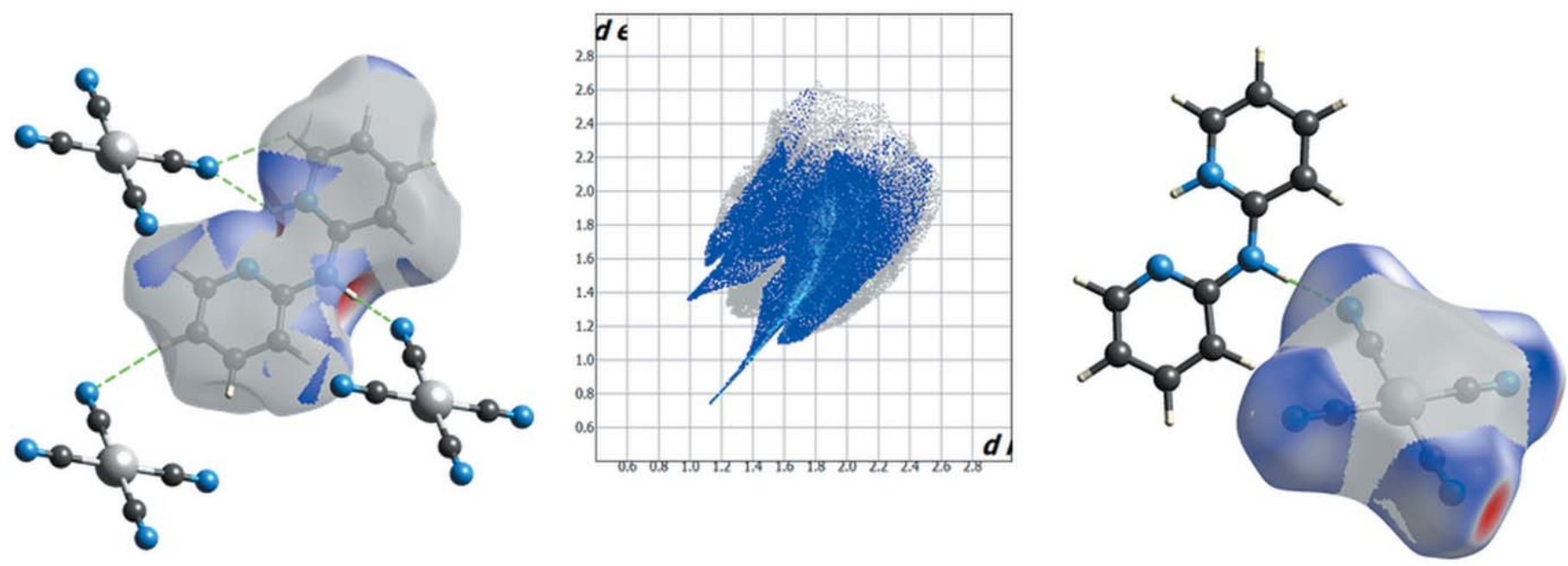

\section{N...H/H...N}
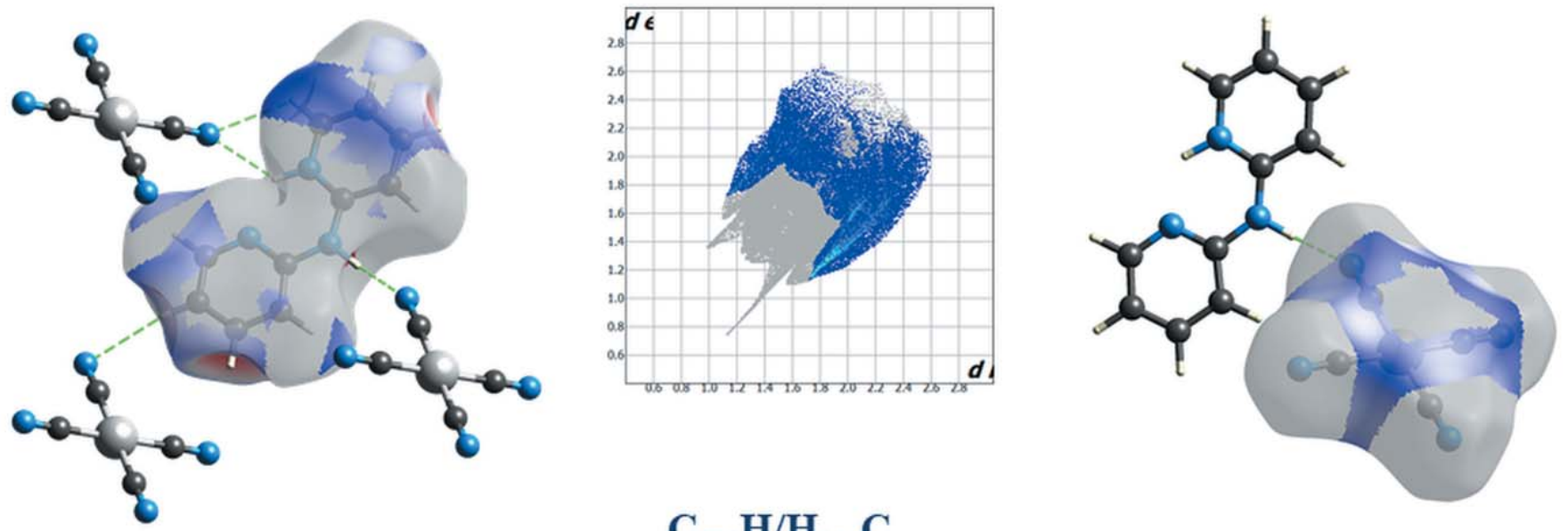

\section{C...H/H...C}
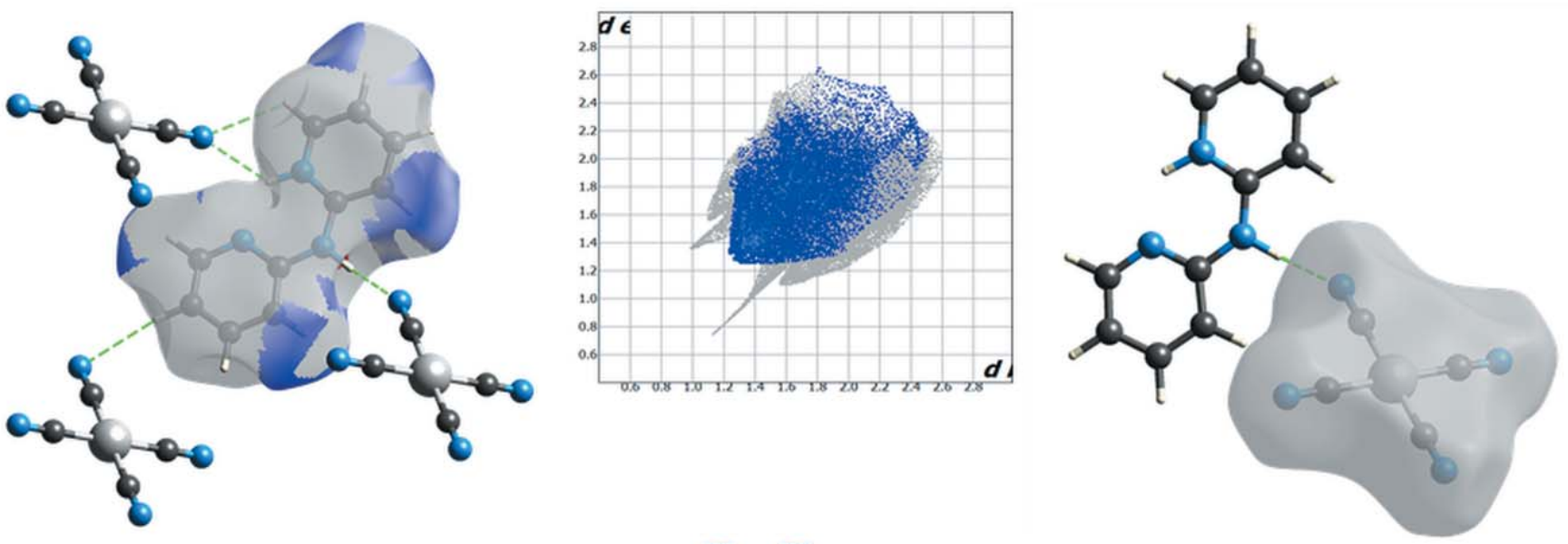

\section{H...H}

Figure 5

Two-dimensional fingerprint plots and relative contributions for (I) resolved into all, $\mathrm{N} \cdots \mathrm{H}, \mathrm{C} \cdots \mathrm{H}$ and $\mathrm{H} \cdots \mathrm{H}$ contacts. 
Table 2

Experimental details.

\begin{tabular}{|c|c|}
\hline \multicolumn{2}{|l|}{ Crystal data } \\
\hline Chemical formula & $\left(\mathrm{C}_{10} \mathrm{H}_{10} \mathrm{~N}_{3}\right)_{2}\left[\mathrm{Ni}(\mathrm{CN})_{4}\right]$ \\
\hline$M_{\mathrm{r}}$ & 507.21 \\
\hline Crystal system, space group & Triclinic, $P \overline{1}$ \\
\hline Temperature $(\mathrm{K})$ & 273 \\
\hline$a, b, c(\AA)$ & 7.1046 (4), 9.1467 (4), $9.3833(4)$ \\
\hline$\alpha, \beta, \gamma\left(^{\circ}\right)$ & $100.182(2), 98.729(2), 97.444(2)$ \\
\hline$V\left(\AA^{3}\right)$ & $585.49(5)$ \\
\hline$Z$ & 1 \\
\hline Radiation type & Mo $K \alpha$ \\
\hline$\mu\left(\mathrm{mm}^{-1}\right)$ & 0.86 \\
\hline Crystal size $(\mathrm{mm})$ & $0.35 \times 0.23 \times 0.19$ \\
\hline \multicolumn{2}{|l|}{ Data collection } \\
\hline Diffractometer & $\begin{array}{l}\text { Oxford Diffraction Xcalibur with } \\
\text { Sapphire CCD detector }\end{array}$ \\
\hline Absorption correction & $\begin{array}{l}\text { Multi-scan (CrysAlis RED; Oxford } \\
\text { Diffraction, 2009) }\end{array}$ \\
\hline$T_{\min }, T_{\max }$ & $0.914,0.962$ \\
\hline $\begin{array}{l}\text { No. of measured, independent and } \\
\text { observed }[I>2 \sigma(I)] \text { reflections }\end{array}$ & $16272,3572,2659$ \\
\hline$R_{\text {int }}$ & 0.052 \\
\hline$(\sin \theta / \lambda)_{\max }\left(\AA^{-1}\right)$ & 0.715 \\
\hline \multicolumn{2}{|l|}{ Refinement } \\
\hline$R\left[F^{2}>2 \sigma\left(F^{2}\right)\right], w R\left(F^{2}\right), S$ & $0.048,0.134,1.07$ \\
\hline No. of reflections & 3572 \\
\hline No. of parameters & 161 \\
\hline $\mathrm{H}$-atom treatment & $\mathrm{H}$-atom parameters constrained \\
\hline$\Delta \rho_{\max }, \Delta \rho_{\min }\left(\mathrm{e} \AA^{-3}\right)$ & $1.01,-0.34$ \\
\hline
\end{tabular}

Computer programs: CrysAlis CCD and CrysAlis RED (Oxford Diffraction, 2009), SHELXS97 (Sheldrick, 2015a), SHELXL2014/7 (Sheldrick, 2015b), DIAMOND (Brandenburg, 2006) and publCIF (Westrip, 2010).

\section{References}

Addala, A., Setifi, F., Kottrup, K. G., Glidewell, C., Setifi, Z., Smith, G. \& Reedijk, J. (2015). Polyhedron, 87, 307-310.

Benmansour, S., Atmani, C., Setifi, F., Triki, S., Marchivie, M. \& Gómez-García, C. J. (2010). Polyhedron, 254, 1468-1478.
Benmansour, S., Setifi, F., Triki, S. \& Gómez-García, C. J. (2012). Inorg. Chem. 51, 2359-2365.

Bowes, K. F., Ferguson, G., Lough, A. J. \& Glidewell, C. (2003). Acta Cryst. B59, 100-117.

Brandenburg, K. (2006). DIAMOND. Crystal Impact GbR, Bonn, Germany.

Bretosh, K., Béreau, V., Duhayon, C., Pichon, C. \& Sutter, J.-P. (2020). Inorg. Chem. Front. 7, 1503-1511.

Ferlay, S., Mallah, T., Ouahès, R., Veillet, P. \& Verdaguer, M. A. (1995). Nature, 378, 701-703.

Groom, C. R., Bruno, I. J., Lightfoot, M. P. \& Ward, S. C. (2016). Acta Cryst. B72, 171-179.

Karaağaç, D., Kürkçüoğlu, G. S., Yeşilel, O. Z., Hökelek, T. \& Süzen, Y. (2013). Inorg. Chim. Acta, 406, 73-80.

Kucheriv, O. I., Shylin, S. I., Ksenofontov, V., Dechert, S., Haukka, M., Fritsky, I. O. \& Gural'skiy, I. A. (2016). Inorg. Chem. 55, 49064914.

McKinnon, J. J., Jayatilaka, D. \& Spackman, M. A. (2007). Chem. Commun. pp. 3814-3816.

Oxford Diffraction (2009). CrysAlis CCD and CrysAlis RED. Oxford Diffraction Ltd, Abingdon, England.

Paharová, J., Černák, J., Boča, R. \& Žák, Z. (2003). Inorg. Chim. Acta, 346, 25-31.

Setifi, F., Benmansour, S., Marchivie, M., Dupouy, G., Triki, S., SalaPala, J., Salaün, J.-Y., Gómez-García, C. J., Pillet, S., Lecomte, C. \& Ruiz, E. (2009). Inorg. Chem. 48, 1269-1271.

Setifi, F., Charles, C., Houille, S., Thétiot, F., Triki, S., Gómez-García, C. J. \& Pillet, S. (2013). Polyhedron, 61, 242-247.

Setifi, F., Milin, E., Charles, C., Thétiot, F., Triki, S. \& Gómez-García, C. J. (2014). Inorg. Chem. 53, 97-104.

Sheldrick, G. M. (2015a). Acta Cryst. A71, 3-8.

Sheldrick, G. M. (2015b). Acta Cryst. C71, 3-8.

Spackman, M. A. \& Jayatilaka, D. (2009). CrystEngComm, 11, 19-32.

Turner, M. J., McKinnon, J. J., Wolff, S. K., Grimwood, D. J., Spackman, P. R., Jayatilaka, D. \& Spackman, M. A. (2017). CrystalExplorer17. University of Western Australia.

Westrip, S. P. (2010). J. Appl. Cryst. 43, 920-925.

Willett, R. D. (1995). Acta Cryst. C51, 1517-1519.

Yoon, J. H., Ryu, D. W., Choi, S. Y., Kim, H. C., Koh, E. K., Tao, J. \& Hong, C. S. (2011). Chem. Commun. 47, 10416-10418.

Yuste, C., Bentama, A., Marino, N., Armentano, D., Setifi, F., Triki, S., Lloret, F. \& Julve, M. (2009). Polyhedron, 28, 1287-1294. 


\section{supporting information}

Acta Cryst. (2020). E76, 1794-1798 [https://doi.org/10.1107/S205698902001419X]

Synthesis, crystal structure and Hirshfeld surface analysis of bis\{2-[(pyridin-2yl)amino] pyridinium\} tetracyanonickelate(II)

\section{Zouaoui Setifi, Hela Ferjani, Fatima Setifi, Safa Ezzine and Mohammed Hadi Al-Douh}

Computing details

Data collection: CrysAlis CCD (Oxford Diffraction, 2009); cell refinement: CrysAlis RED (Oxford Diffraction, 2009); data reduction: CrysAlis RED (Oxford Diffraction, 2009); program(s) used to solve structure: SHELXS97 (Sheldrick, 2015a); program(s) used to refine structure: SHELXL2014/7 (Sheldrick, 2015b); molecular graphics: DIAMOND (Brandenburg, 2006); software used to prepare material for publication: publCIF (Westrip, 2010).

(I)

\section{Crystal data}

$\left(\mathrm{C}_{10} \mathrm{H}_{10} \mathrm{~N}_{3}\right)_{2}\left[\mathrm{Ni}(\mathrm{CN})_{4}\right]$

$M_{r}=507.21$

Triclinic, $P \overline{1}$

$a=7.1046(4) \AA$

$b=9.1467(4) \AA$

$c=9.3833(4) \AA$

$\alpha=100.182(2)^{\circ}$

$\beta=98.729(2)^{\circ}$

$\gamma=97.444(2)^{\circ}$

$V=585.49(5) \AA^{3}$

$$
\begin{aligned}
& Z=1 \\
& F(000)=262 \\
& D_{\mathrm{x}}=1.439 \mathrm{Mg} \mathrm{m}^{-3}
\end{aligned}
$$

Mo $K \alpha$ radiation, $\lambda=0.71073 \AA$

Cell parameters from 7173 reflections

$\theta=2.8-27.9^{\circ}$

$\mu=0.86 \mathrm{~mm}^{-1}$

$T=273 \mathrm{~K}$

Plate, pale yellow

$0.35 \times 0.23 \times 0.19 \mathrm{~mm}$

\section{Data collection}

Oxford Diffraction Xcalibur Sapphire CCD detector diffractometer

Radiation source: Enhance (Mo) X-ray Source $\omega$ scans

Absorption correction: multi-scan

(CrysAlis RED; Oxford Diffraction, 2009)

$T_{\min }=0.914, T_{\max }=0.962$

16272 measured reflections

3572 independent reflections

2659 reflections with $I>2 \sigma(I)$

$R_{\text {int }}=0.052$

$\theta_{\text {max }}=30.6^{\circ}, \theta_{\min }=2.2^{\circ}$

$h=-10 \rightarrow 10$

$k=-13 \rightarrow 13$

$l=-13 \rightarrow 13$

Refinement

Refinement on $F^{2}$

Least-squares matrix: full

$R\left[F^{2}>2 \sigma\left(F^{2}\right)\right]=0.048$

$w R\left(F^{2}\right)=0.134$

$S=1.07$

3572 reflections

161 parameters

0 restraints

Primary atom site location: structure-invariant direct methods

Hydrogen site location: inferred from neighbouring sites

$\mathrm{H}$-atom parameters constrained

$w=1 /\left[\sigma^{2}\left(F_{\mathrm{o}}^{2}\right)+(0.0546 P)^{2}+0.3025 P\right]$

where $P=\left(F_{\mathrm{o}}{ }^{2}+2 F_{\mathrm{c}}{ }^{2}\right) / 3$

$(\Delta / \sigma)_{\max }<0.001$

$\Delta \rho_{\max }=1.01 \mathrm{e} \AA^{-3}$ 
$\Delta \rho_{\min }=-0.34$ e $\AA^{-3}$
Extinction correction: SHELXL-2014/7

(Sheldrick 2014,

$\mathrm{Fc}^{*}=\mathrm{kFc}\left[1+0.001 \mathrm{xFc}^{2} \lambda^{3} / \sin (2 \theta)\right]^{-1 / 4}$

Extinction coefficient: 0.091 (17)

Special details

Geometry. All esds (except the esd in the dihedral angle between two 1.s. planes) are estimated using the full covariance matrix. The cell esds are taken into account individually in the estimation of esds in distances, angles and torsion angles; correlations between esds in cell parameters are only used when they are defined by crystal symmetry. An approximate (isotropic) treatment of cell esds is used for estimating esds involving l.s. planes.

Fractional atomic coordinates and isotropic or equivalent isotropic displacement parameters $\left(\AA^{2}\right)$

\begin{tabular}{|c|c|c|c|c|}
\hline & $x$ & $y$ & $z$ & $U_{\text {iso }} * / U_{\text {eq }}$ \\
\hline Nil & 0.5000 & 0.0000 & 0.0000 & 0.04195 (19) \\
\hline $\mathrm{C} 1$ & $0.6743(4)$ & $0.0656(3)$ & $0.1772(3)$ & $0.0508(6)$ \\
\hline N1 & $0.7800(5)$ & $0.1080(3)$ & $0.2861(3)$ & $0.0745(8)$ \\
\hline $\mathrm{C} 2$ & $0.3639(4)$ & $0.1552(3)$ & $0.0568(2)$ & $0.0455(5)$ \\
\hline $\mathrm{N} 2$ & $0.2831(4)$ & $0.2503(3)$ & $0.0942(3)$ & $0.0624(6)$ \\
\hline N4 & $0.1820(3)$ & $0.7199(2)$ & $0.3981(2)$ & $0.0445(4)$ \\
\hline $\mathrm{H} 4 \mathrm{~A}$ & 0.1949 & 0.7110 & 0.4886 & $0.053^{*}$ \\
\hline N5 & $0.2473(3)$ & $0.5418(2)$ & $0.5852(2)$ & $0.0484(5)$ \\
\hline N3 & $0.2311(3)$ & $0.4715(2)$ & $0.3342(2)$ & $0.0492(5)$ \\
\hline $\mathrm{H} 3 \mathrm{~A}$ & 0.2354 & 0.3997 & 0.2625 & $0.059 *$ \\
\hline $\mathrm{C} 3$ & $0.1977(3)$ & $0.6028(3)$ & 0.2949 (3) & $0.0422(5)$ \\
\hline $\mathrm{C} 8$ & $0.2592(3)$ & $0.4356(3)$ & $0.4722(3)$ & $0.0443(5)$ \\
\hline $\mathrm{C} 5$ & $0.1462(4)$ & $0.8522(3)$ & $0.3626(3)$ & $0.0508(6)$ \\
\hline H5 & 0.1351 & 0.9313 & 0.4365 & $0.061 *$ \\
\hline $\mathrm{C} 4$ & $0.1807(4)$ & $0.6192(3)$ & 0.1479 (3) & $0.0503(6)$ \\
\hline $\mathrm{H} 4$ & 0.1942 & 0.5397 & 0.0753 & $0.060^{*}$ \\
\hline $\mathrm{C} 12$ & $0.3276(4)$ & $0.2629(3)$ & $0.6262(4)$ & $0.0595(7)$ \\
\hline $\mathrm{H} 12$ & 0.3538 & 0.1690 & 0.6407 & $0.071 *$ \\
\hline $\mathrm{C} 7$ & $0.1442(4)$ & $0.7522(3)$ & $0.1118(3)$ & $0.0557(6)$ \\
\hline $\mathrm{H} 7$ & 0.1314 & 0.7635 & 0.0144 & $0.067 *$ \\
\hline $\mathrm{C} 10$ & $0.2763(4)$ & $0.5097(3)$ & $0.7197(3)$ & $0.0553(6)$ \\
\hline $\mathrm{H} 10$ & 0.2695 & 0.5836 & 0.7998 & $0.066^{*}$ \\
\hline C6 & $0.1264(4)$ & $0.8713(3)$ & $0.2224(3)$ & $0.0564(7)$ \\
\hline H6 & 0.1013 & 0.9625 & 0.1994 & $0.068^{*}$ \\
\hline C9 & $0.3006(4)$ & $0.2930(3)$ & 0.4869 (3) & $0.0524(6)$ \\
\hline H9 & 0.3096 & 0.2214 & 0.4055 & $0.063^{*}$ \\
\hline C11 & $0.3157(4)$ & $0.3727(4)$ & 0.7447 (3) & $0.0591(7)$ \\
\hline H11 & 0.3341 & 0.3540 & 0.8397 & $0.071 *$ \\
\hline
\end{tabular}

Atomic displacement parameters $\left(\AA^{2}\right)$

\begin{tabular}{lllllll}
\hline & $U^{11}$ & $U^{22}$ & $U^{33}$ & $U^{12}$ & $U^{13}$ & $U^{23}$ \\
\hline Ni1 & $0.0617(3)$ & $0.0339(2)$ & $0.0330(2)$ & $0.01697(18)$ & $0.01155(18)$ & $0.00464(15)$ \\
C1 & $0.0751(18)$ & $0.0380(12)$ & $0.0419(12)$ & $0.0238(11)$ & $0.0105(12)$ & $0.0040(9)$ \\
N1 & $0.100(2)$ & $0.0626(15)$ & $0.0527(14)$ & $0.0317(15)$ & $-0.0091(14)$ & $-0.0053(12)$
\end{tabular}




\begin{tabular}{lllllll} 
C2 & $0.0628(15)$ & $0.0412(12)$ & $0.0328(10)$ & $0.0168(11)$ & $0.0073(10)$ & $0.0028(9)$ \\
N2 & $0.0820(17)$ & $0.0554(13)$ & $0.0494(12)$ & $0.0333(12)$ & $0.0076(12)$ & $-0.0032(10)$ \\
N4 & $0.0475(11)$ & $0.0447(10)$ & $0.0424(10)$ & $0.0101(8)$ & $0.0080(8)$ & $0.0096(8)$ \\
N5 & $0.0520(12)$ & $0.0482(11)$ & $0.0468(11)$ & $0.0092(9)$ & $0.0106(9)$ & $0.0115(9)$ \\
N3 & $0.0664(14)$ & $0.0403(10)$ & $0.0421(10)$ & $0.0166(9)$ & $0.0133(10)$ & $0.0022(8)$ \\
C3 & $0.0382(12)$ & $0.0411(11)$ & $0.0485(12)$ & $0.0077(9)$ & $0.0070(9)$ & $0.0123(9)$ \\
C8 & $0.0402(12)$ & $0.0451(12)$ & $0.0495(13)$ & $0.0045(9)$ & $0.0081(10)$ & $0.0160(10)$ \\
C5 & $0.0544(15)$ & $0.0406(12)$ & $0.0582(15)$ & $0.0106(10)$ & $0.0111(12)$ & $0.0090(11)$ \\
C4 & $0.0538(15)$ & $0.0525(14)$ & $0.0450(13)$ & $0.0137(11)$ & $0.0095(11)$ & $0.0069(10)$ \\
C12 & $0.0574(16)$ & $0.0509(15)$ & $0.0743(19)$ & $0.0069(12)$ & $0.0059(14)$ & $0.0294(14)$ \\
C7 & $0.0589(16)$ & $0.0636(16)$ & $0.0505(14)$ & $0.0141(13)$ & $0.0117(12)$ & $0.0228(12)$ \\
C10 & $0.0572(16)$ & $0.0640(16)$ & $0.0456(13)$ & $0.0076(13)$ & $0.0120(12)$ & $0.0126(12)$ \\
C6 & $0.0593(16)$ & $0.0494(14)$ & $0.0670(17)$ & $0.0147(12)$ & $0.0115(13)$ & $0.0242(13)$ \\
C9 & $0.0585(16)$ & $0.0417(12)$ & $0.0576(15)$ & $0.0091(11)$ & $0.0097(12)$ & $0.0109(11)$ \\
C11 & $0.0514(15)$ & $0.0747(19)$ & $0.0545(15)$ & $0.0034(13)$ & $0.0068(12)$ & $0.0291(14)$ \\
& & & & & & \\
\hline
\end{tabular}

Geometric parameters $\left(A,{ }^{\circ}\right)$

\begin{tabular}{|c|c|c|c|}
\hline $\mathrm{Ni} 1-\mathrm{C} 2$ & $1.865(2)$ & $\mathrm{C} 8-\mathrm{C} 9$ & $1.399(3)$ \\
\hline $\mathrm{Ni} 1-\mathrm{C} 2^{\mathrm{i}}$ & $1.865(2)$ & $\mathrm{C} 5-\mathrm{C} 6$ & $1.346(4)$ \\
\hline $\mathrm{Ni1}-\mathrm{C} 1$ & $1.867(3)$ & $\mathrm{C} 5-\mathrm{H} 5$ & 0.9300 \\
\hline $\mathrm{Ni} 1-\mathrm{C}^{\mathrm{i}}$ & $1.867(3)$ & $\mathrm{C} 4-\mathrm{C} 7$ & $1.365(4)$ \\
\hline $\mathrm{C} 1-\mathrm{N} 1$ & $1.145(4)$ & $\mathrm{C} 4-\mathrm{H} 4$ & 0.9300 \\
\hline $\mathrm{C} 2-\mathrm{N} 2$ & $1.136(3)$ & $\mathrm{C} 12-\mathrm{C} 9$ & $1.373(4)$ \\
\hline $\mathrm{N} 4-\mathrm{C} 3$ & $1.340(3)$ & $\mathrm{C} 12-\mathrm{C} 11$ & $1.381(4)$ \\
\hline N4-C5 & $1.355(3)$ & $\mathrm{C} 12-\mathrm{H} 12$ & 0.9300 \\
\hline $\mathrm{N} 4-\mathrm{H} 4 \mathrm{~A}$ & 0.8600 & $\mathrm{C} 7-\mathrm{C} 6$ & $1.399(4)$ \\
\hline N5-C8 & $1.326(3)$ & $\mathrm{C} 7-\mathrm{H} 7$ & 0.9300 \\
\hline $\mathrm{N} 5-\mathrm{C} 10$ & $1.337(3)$ & $\mathrm{C} 10-\mathrm{C} 11$ & $1.371(4)$ \\
\hline $\mathrm{N} 3-\mathrm{C} 3$ & $1.355(3)$ & $\mathrm{C} 10-\mathrm{H} 10$ & 0.9300 \\
\hline N3-C8 & $1.383(3)$ & $\mathrm{C} 6-\mathrm{H} 6$ & 0.9300 \\
\hline $\mathrm{N} 3-\mathrm{H} 3 \mathrm{~A}$ & 0.8600 & C9- $\mathrm{H} 9$ & 0.9300 \\
\hline $\mathrm{C} 3-\mathrm{C} 4$ & $1.402(3)$ & $\mathrm{C} 11-\mathrm{H} 11$ & 0.9300 \\
\hline $\mathrm{C} 2-\mathrm{Ni} 1-\mathrm{C} 2^{\mathrm{i}}$ & 180.0 & $\mathrm{~N} 4-\mathrm{C} 5-\mathrm{H} 5$ & 119.4 \\
\hline $\mathrm{C} 2-\mathrm{Ni} 1-\mathrm{C} 1$ & $89.06(10)$ & $\mathrm{C} 7-\mathrm{C} 4-\mathrm{C} 3$ & $119.8(2)$ \\
\hline $\mathrm{C} 2 \mathrm{i}-\mathrm{Ni} 1-\mathrm{C} 1$ & $90.94(10)$ & $\mathrm{C} 7-\mathrm{C} 4-\mathrm{H} 4$ & 120.1 \\
\hline $\mathrm{C} 2-\mathrm{Ni} 1-\mathrm{C}^{\mathrm{i}}$ & $90.94(10)$ & $\mathrm{C} 3-\mathrm{C} 4-\mathrm{H} 4$ & 120.1 \\
\hline $\mathrm{C} 2^{\mathrm{i}}-\mathrm{Ni} 1-\mathrm{C} 1^{\mathrm{i}}$ & $89.06(10)$ & $\mathrm{C} 9-\mathrm{C} 12-\mathrm{C} 11$ & $119.7(3)$ \\
\hline $\mathrm{C} 1-\mathrm{Ni} 1-\mathrm{C}^{\mathrm{i}}$ & 180.0 & $\mathrm{C} 9-\mathrm{C} 12-\mathrm{H} 12$ & 120.2 \\
\hline $\mathrm{N} 1-\mathrm{C} 1-\mathrm{Ni1}$ & $178.8(2)$ & $\mathrm{C} 11-\mathrm{C} 12-\mathrm{H} 12$ & 120.2 \\
\hline $\mathrm{N} 2-\mathrm{C} 2-\mathrm{Ni} 1$ & $178.6(2)$ & $\mathrm{C} 4-\mathrm{C} 7-\mathrm{C} 6$ & $119.5(2)$ \\
\hline $\mathrm{C} 3-\mathrm{N} 4-\mathrm{C} 5$ & $121.3(2)$ & $\mathrm{C} 4-\mathrm{C} 7-\mathrm{H} 7$ & 120.3 \\
\hline $\mathrm{C} 3-\mathrm{N} 4-\mathrm{H} 4 \mathrm{~A}$ & 119.4 & $\mathrm{C} 6-\mathrm{C} 7-\mathrm{H} 7$ & 120.3 \\
\hline $\mathrm{C} 5-\mathrm{N} 4-\mathrm{H} 4 \mathrm{~A}$ & 119.4 & $\mathrm{~N} 5-\mathrm{C} 10-\mathrm{C} 11$ & $122.9(3)$ \\
\hline $\mathrm{C} 8-\mathrm{N} 5-\mathrm{C} 10$ & $117.8(2)$ & $\mathrm{N} 5-\mathrm{C} 10-\mathrm{H} 10$ & 118.5 \\
\hline $\mathrm{C} 3-\mathrm{N} 3-\mathrm{C} 8$ & $129.7(2)$ & $\mathrm{C} 11-\mathrm{C} 10-\mathrm{H} 10$ & 118.5 \\
\hline $\mathrm{C} 3-\mathrm{N} 3-\mathrm{H} 3 \mathrm{~A}$ & 115.1 & $\mathrm{C} 5-\mathrm{C} 6-\mathrm{C} 7$ & $119.1(2)$ \\
\hline
\end{tabular}




$\mathrm{C} 8-\mathrm{N} 3-\mathrm{H} 3 \mathrm{~A}$
$\mathrm{~N} 4-\mathrm{C} 3-\mathrm{N} 3$
$\mathrm{~N} 4-\mathrm{C} 3-\mathrm{C} 4$
$\mathrm{~N} 3-\mathrm{C} 3-\mathrm{C} 4$
$\mathrm{~N} 5-\mathrm{C} 8-\mathrm{N} 3$
$\mathrm{~N} 5-\mathrm{C} 8-\mathrm{C} 9$
$\mathrm{~N} 3-\mathrm{C} 8-\mathrm{C} 9$
$\mathrm{C} 6-\mathrm{C} 5-\mathrm{N} 4$
$\mathrm{C} 6-\mathrm{C} 5-\mathrm{H} 5$

115.1

$119.7(2)$

$119.0(2)$

$121.3(2)$

$117.0(2)$

$123.4(2)$

$119.6(2)$

$121.3(2)$

119.4
$\mathrm{C} 5-\mathrm{C} 6-\mathrm{H} 6$

$\mathrm{C} 7-\mathrm{C} 6-\mathrm{H} 6$

$\mathrm{C} 12-\mathrm{C} 9-\mathrm{C} 8$

C12- $9-\mathrm{H} 9$

$\mathrm{C} 8-\mathrm{C} 9-\mathrm{H} 9$

$\mathrm{C} 10-\mathrm{C} 11-\mathrm{C} 12$

$\mathrm{C} 10-\mathrm{C} 11-\mathrm{H} 11$

C12-C11-H11
120.4

120.4

$117.4(3)$

121.3

121.3

$118.8(3)$

120.6

120.6

Symmetry code: (i) $-x+1,-y,-z$.

Hydrogen-bond geometry $\left(\AA,{ }^{\circ}\right)$

\begin{tabular}{lllll}
\hline$D-\mathrm{H} \cdots A$ & $D-\mathrm{H}$ & $\mathrm{H} \cdots A$ & $D \cdots A$ & $D-\mathrm{H} \cdots A$ \\
\hline $\mathrm{N} 3-\mathrm{H} 3 A \cdots \mathrm{N} 2$ & 0.86 & 2.00 & $2.853(3)$ & 172 \\
$\mathrm{~N} 4-\mathrm{H} 4 A \cdots \mathrm{N} 5$ & 0.86 & 1.97 & $2.629(3)$ & 132 \\
$\mathrm{~N} 4-\mathrm{H} 4 A \cdots \mathrm{N} 1^{\mathrm{ii}}$ & 0.86 & 2.41 & $3.055(3)$ & 132 \\
$\mathrm{C} 5-\mathrm{H} 5 \cdots \mathrm{N} 1^{\mathrm{ii}}$ & 0.93 & 2.68 & $3.206(4)$ & 117 \\
\hline
\end{tabular}

Symmetry code: (ii) $-x+1,-y+1,-z+1$. 